\title{
THE ROLE OF EPITHELIAL AND VASCULAR-ENDOTHELIAL CADHERIN IN THE DIFFERENTIATION AND MAINTANCE OF TISSUE INTEGRITY
}

\begin{abstract}
Petr Nachtigal, Andrea Gojová, Vladimír Semecký
Charles University in Prague, Faculty of Pharmacy in Hradec Králové: Department of Biological and Medical Sciences

Summary: The present review has focused on the cell adhesion molecules from the cadherin superfamily, in particular on E- and VE-cadherin. In general, cadherins are a large group of cell adhesion molecules located at intercellular junctions called adherent junctions. They play an important role in embryogenesis and morphogenesis in animals and humans due to their adhesive and cell-signalling functions. Disturbances of the expression or function of cadherins and their associated proteins called catenins are crucial for the initiation and development of many pathological states. E-cadherin is an epithelium-specific cadherin that is required for the development and maintenance of the normal function of all epithelial cells in tissues. The loss or down-regulation of E-cadherin is a key event in the process of tumour invasion and metastasis. The assessment of E-cadherin immunoreactivity may be a useful prognostic marker in some cancers, complementary to the established prognostic factors. VE-cadherin is an endothelium-specific cadherin, which plays a relevant role in vascular homeostasis. It has been demonstrated that VE-cadherin is required for normal vasculogenesis, angiogenesis, and for the maintenance of vascular integrity. Disruption of VE-cadherin-catenin complexes by some inflammatory agents such as thrombin, by inflammatory cells, or shear stress is accompanied by an increase in vascular permeability in vivo and in vitro.
\end{abstract}

Key words: Cadherin family; E-cadherin; VE-cadherin; Cancerogenesis; Endothelial permeability

\section{Introduction}

Cell adhesion molecules are substances of a protein character that are necessary for normal embryogenesis, morphogenesis, tissue formation and reparation, but they are also involved in many pathophysiological processes such as inflammation, angiogenesis, thrombosis, tumour invasion and metastasis (19). At present, there are four main classes of cytoadhesion molecules: the immunoglobulin group of adhesion molecules, cadherins, selectins, and integrins (22). In the present review the authors concentrate on cell adhesion molecules from the cadherin family.

The cadherins are a family of transmembrane glycoproteins that mediate adhesion through a $\mathrm{Ca}^{2+}$ dependent mechanism. Cadherins are usually localized at intercellular junctions called adherens junctions (41). The cadherins form a superfamily with at least six subfamilies, which can be distinguished on the basis of the protein domain composition, genomic structure, and phylogenetic analysis of the protein sequences. These subfamilies comprise classical or type-I cadherins (E-, N-, R-, P-cadherin), atypical or type-II cadherins (Cadherin-6, -7, -8, -10), desmosomal cadherins (desmocollins, desmogleins), protocadherins (Protocadherin-1, -2, OL-protocadherin, CNR protocadherins), and Flamingo cadherins (25).

\section{Structural properties of the cadherin superfamily}

In this review the present authors have aimed at the structure of classical cadherins. The cadherins consist of an extracellular adhesive domain, transmembrane segment, and cytoplasmic domain (5). The extracellular domain is responsible for cell-to-cell adhesion between cadherins. It is composed of four or five repeated $\mathrm{Ca}^{2+}$ binding subdomains of about 110 amino acids (39). The structure of this domain is determined by the binding of calcium ions, and the presence of $\mathrm{Ca}^{2+}$ ions is also necessary for the adhesion with cadherin molecules on neighbouring cells. Other classes of adhesion molecules, e.g., members of the Ig superfamily, do not depend on $\mathrm{Ca}^{2+}$ for their adhesive function (29). The adhesive interface is located at the most aminoterminal repeat. Structural data suggest that the extracellular domains form lateral dimers on the cell surface which interact with dimers from the opposing cells to create a zipper-like structure (34). Highly conserved cytoplasmic domains interact with cytosolic proteins from "armadillo family" called catenins. It has been demonstrated that there are three main catenins in cadherin-catenin complexes that provide the linkage of cadherin to the actin cytoskeleton (18). $\beta$-Catenin and plakoglobin (also called $\gamma$-catenin), interact with $\alpha$-catenin that provides connection with the 
actin cytoskeleton. Other catenins can also be associated with the cytoplasmic domain of cadherins, including different isoforms of the so-called $\mathrm{p} 120^{\mathrm{ctn}}$. It has been found that this catenin interacts with a membrane-proximal region of cadherin that has been shown to be responsible for lateral clustering. This suggest that $\mathrm{p} 120^{\mathrm{ctn}}$ can regulate the strength of cadherin-mediated adhesion (43). In general, the interaction of cytoplasmic domain with the actin cytoskeleton via catenins significantly increases the strength of the intercellular junctions (45).

\section{Function of the cadherins}

There are two main functional features of cadherins. They are responsible for cell-to-cell adhesion and they have a very important cell signalling function. Cadherins mediate homotypic interactions by binding to their homologues on an adjacent cell. However, it has been found that some members of this family can provide heterotypic interactions, but this is not the predominant adhesion mechanism (27). There are several ways of how cadherin could be involved in cell-signalling events. First, through their homotypic binding they can approach the opposing membranes of neighbouring cells in close proximity and enable interactions of ligands and receptors of these opposing cells and stimulate juxtacrine signalling. Second, because cadherins are able to control the polarity of cells they can affect sig- nalling via their influence on the distribution of membrane proteins, including transmembrane receptors. Finally, cadherins may behave as ligands or receptors and hence they have direct cell signalling activity (18). All these signalling functions of cadherins are associated with the activation of some regulatory cascade comprising the action of tyrosin kinases and tyrosin phosphatases. Cytoplasmic domains of cadherin, $\beta$-catenin and plakoglobin are common targets of these regulatory proteins (38). Adhesive and signalling properties of cadherins can not be separated. Any changes in the expression or function of cadherins might lead to initiation or progression of pathological processes. Within many members of the cadherin superfamily, the present authors have focused on two extensively studied ones, E-cadherin and VE-cadherin.

\section{Epithelial cadherin (E-cadherin, LCAM, ovomorulin)}

E-cadherin is an epithelium-specific cadherin. This cadherin is a member of the classical cadherin subfamily. Normal expression and function of E-cadherin is required for proper embryogenesis and morphogenesis of various tissues. Variations in E-cadherin expression have been noted during specific events in embryonic morphogenesis (30). Mutation of the E-cadherin gene leads to early embryonic lethality which is proceeded by a loss of cell-to-cell adhe-

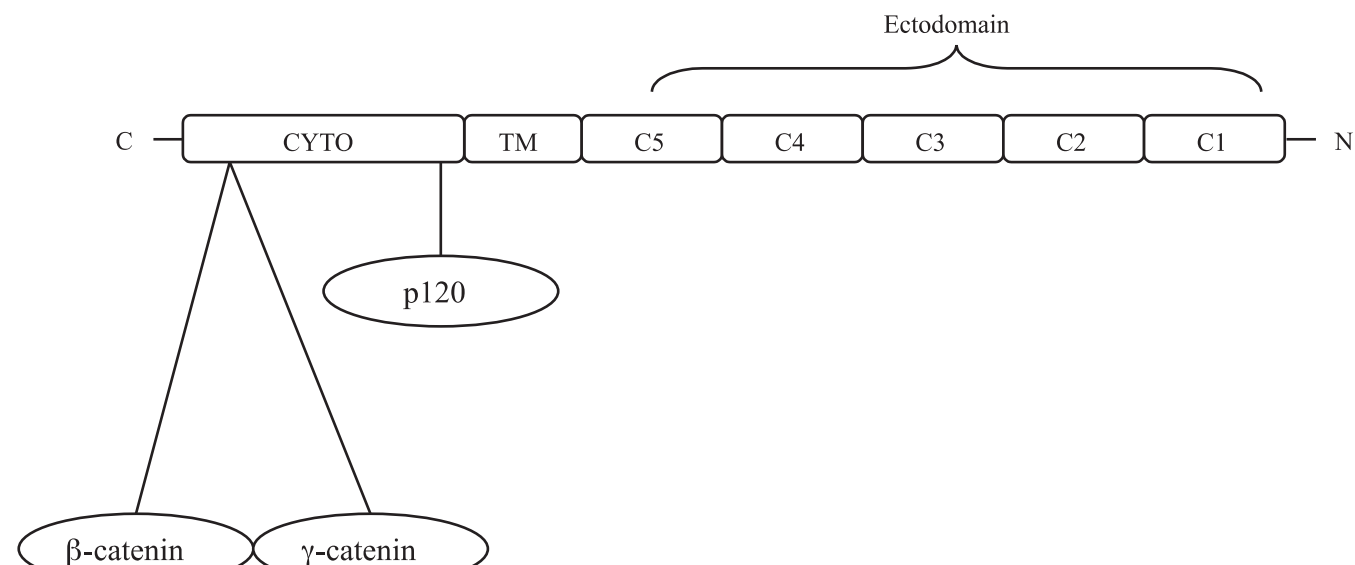

Fig. 1: Structure of cadherin-catenin-complex.

Representation of cadherin-catenin complex depicting cadherin ectodomain, transmembrane region (TM) and carboxy-terminal cytodomain (CYTO) and catenins that link cadherin to the cytoskeleton. Ectodamain of classical cadherins consists of five repeated domains (C1-C5) with adhesive interface located at $\mathrm{C} 1$ domain. Carboxy-terminal cytodomain has binding sites for $\beta$-catenin and $\gamma$-catenin (plakoglobin) and membrane proximal binding site for p120.

$\beta$-catenin and $\gamma$-catenin are associated with $\alpha$-catenin that provide linkage to actin cytoskeleton. 
sion at the morula stage (5). E-cadherin is necessary during normal neural development (35). It has been demonstrated to occur locally and persistently in the murine central and peripheral nervous system during neural development (36). Expression of E-cadherin (together with P-and N-cadherin) has been observed in murine primordial germ cells. E-cadherin is concentrated at the sites of cell-to-cell contacts of primordial germ cells (PGCs), suggesting an active role in PGCs-PGCs interaction and recognition (6).

In the past few years, the alteration in expression and function of E-cadherin has been correlated with cancer development (5). Loss or reduction of E-cadherin expression is in relation with enhanced aggressiveness and dedifferentiation of many carcinomas which has been reviewed by Beavon (4). Asgeirsson et al. have shown that decreased E-cadherin expression is frequent in breast cancer and that a loss of E-cadherin expression is associated with a loss of heterozygosity in the infiltrating lobular breast carcinomas but not in the infiltrating ductal carcinomas. Furthermore, the loss of expression of E-cadherin is an important prognostic marker, especially for disease recurrence in nodenegative breast cancer patients and may even be more informative than tumour size or oestrogen receptor expression (3). Ghadimi et al. have shown that reduced expression of E-cadherin and even $\alpha$-catenin is observed in primary colorectal carcinoma. Moreover, they were able to demonstrate a significant correlation between the histopathological grading of the tumours and an increased loss of E-cadherin and $\alpha$-catenin expression. Defective expression was significantly more frequent in less differentiated carcinomas (G3-4) with a pronounced loss of epithelial morphology than in better differentiated tumours (G1/G2) (17). Garcia del Muro et al. have suggested that losses of E-cadherin and $\beta$-catenin as confirmed by immunohistochemistry are important prognostic markers in patients with bladder carcinoma. This leakage of E-cadherin expression was associated with high grade and invasive stage of bladder carcinoma. Further, a loss of E-cadherin expression was a significant prognostic indicator of decreased survival, independent of known prognostic factors such as grade, stage, or p53 status (23). There are many other studies which have described a relation between decreased E-cadherin and/or catenin expression and its correlation with dedifferentiation, infiltrative tumour growth, distant metastasis, and poor survival for patients with gastric carcinoma (37), pancreatic carcinoma $(40,16)$, prostate cancer (12).

Despite the fact that E-cadherin is extensively studied in relation to cancerogenesis, Bobryshev et al. have elucidated expression of E-cadherin in human atherosclerotic lesions. It has been described that E-cadherin is expressed by macrophage origin intimal cells transforming themselves into foam cells, but there were no expression of E-cadherin in non-atherosclerotic intima. They suggested that E-cadherin might be important for foam cell aggregation. If E-cadherin is involved in foam cell aggregation, it might be also invol- ved in the development of the lipid core which is an important step of progression of atherosclerosis (9).

\section{Vascular endothelial cadherin (VE-cadherin, cadherin 5 or 7 B4)}

VE-cadherin is endothelium-specific cadherin and it is located strictly at intercellular junctions (zonulla adherens) of essentially all types of vessels both in vitro and in vivo $(14,15)$. VE-cadherin has been first identified by Lampugnani et al. by adopting an indirect approach of developing mouse mAbs to human endothelial cells (20). In term of the structure, VE-cadherin is composed of an extracellular domain, a transmembrane segment, and a cytoplasmic domain that form complexes with catenins and mediate the association of VE-cadherin with the actin cytoskeleton. However, compared with classical cadherins, the VE-cadherin amino acid sequence shows considerable differences (only 23\% identity when compared with classical cadherins such as E-, N-, and placental (P)-cadherins) $(11,24)$.

VE-cadherin is required for normal vasculogenesis and angiogenesis and for the maintenance of vascular integrity and permeability in adults (13). VE-cadherin is expressed in the embryo at very early stages of vascular development in mesodermal cells of the yolk sac mesenchyme. At later embryonic stages, VE-cadherin expression is restricted to the peripheral layer of blood islands, which gives rise to endothelial cells (10). The role of VE cadherin permeability control is consistent with the observation that the VE-cadherin-catenin complex is the target of the action of permeability-increasing agents. Rabiet et al. have shown that thrombin, which is known to induce profound alterations of endothelial cell monolayer permeability in vitro and in vivo, caused endothelial cell retraction accompanied by a redistribution of VE-cadherin and catenin from adherence junctions. This disassembly of adherence junctions was accompanied by an increase in vascular permeability (28). The proinflammatory cytokines tumour necrosis factor$\alpha$ (TNF- $\alpha)$ and interferon- $\gamma$ (INF- $\gamma$ ) act synergistically in vitro and in vivo to activate the endothelium, resulting in cellular responses such as altered morphology, loss of barrier function, and adhesion molecule upregulation and/or redistribution $(31,32)$. Wond et al. have described that the tumour necrosis factor- $\alpha$ (TNF- $\alpha$ ) and interferon- $\gamma$ (INF- $\gamma$ ), in combination, affect the barrier function of the vascular endothelial lining by direct stimulation of the endothelium that results in the disruption of VE-cadherin mediated cellto-cell adhesion, which is succeeded by an increase in the permeability of mesenteric venules (44). Andriopoulous et al. have studied the effect of histamine, another mediator of inflammatory reaction, on adherens junction organization in cultured endothelial cells. They have reported that histamine induces tyrosine phosphorylation of VE-cadherin and catenins, which results in an increase in endothelial permeability. The effect of histamine was specific for VE-cadherin, there was no phosphorylation in $\mathrm{N}$-cadherin, another 
major endothelial cadherin (2). In addition to agonists described above, endothelial permeability is affected by a group of inflammatory cells, namely polymorphonuclear leukocytes (PMNs) (33). Several groups have shown that activated polymorphonuclear leukocytes (PMNs) dramatically alter molecular composition and organization of VE-cadherin-catenin complexes in endothelial cells. This disruption of VE-cadherin-catenin complexes leads to disassembly of adherens junctions which is followed by an increase in endothelial permeability $(42,1)$. These inflammatory agents and cells are not the only ones that might exert effects on VE-cadherin-catenin complexes. The structure and physiology of endothelial cells are influenced by shear stresses of blood flow. The most obvious structural responses of endothelium to shear stress are changes in the cell shape and orientation; in areas of low or inconsistent shear stress, in vivo or in vitro endothelial cells form a cuboidal, cobblestone shape, whereas they elongate and align in the direction of flow when shear stress is moderate or high (21). Noria et al. have examined transient and steady-state effects of shear stress on the cadherin-catenin complex at endothelial adherens junctions. They have reported that initiation of shear stress on endothelium causes partial disassembly of adherens junctions followed by a reassembly that reflected shear-induced reorganization of actin distribution. After adaptation to shear stress, adherens junction proteins were localized in adhesion plaques (adherens plaques) that were distinct from the linear belt-like distribution that predominates in static cultures. Thus, adherens junctions in the endothelium exposed to physiological levels of shear stress are structurally distinct from such junctions in static endothelial cell cultures or in other epithelial monolayers (26). As mentioned above, the normal expression and function of VE-cadherin is necessary for the maintenance of normal endothelial permeability; however, it is important in vasculogenesis in the embryo as well as in adults. Bobryshev et al. have examined the expression of VE-cadherin in atherosclerotic lesions. They have demonstrated that VE-cadherin is expressed in early sprouts of neocapillaries and it suggests that VE-cadherin is involved in the ingrowth of medial capillaries into the intima (8). This neovascularization is important for local immune-inflammatory reactions in atherosclerotic plaques (7).

\section{Conclusion}

In this review the authors have described structure and function of cell adhesion molecules from cadherin family. We have focused on two extensively studied E-cadherin and VE-cadherin. Both VE-cadherin and E-cadherin are crucial for proper embryogenesis, morphogenesis but they are involved in many pathological states. Changes in expression and function of E-cadherin seems to be important for development of carcinoma in various tissues. VE-cadherin is specific cadherin which is expressed by endothelial cells and plays important role in vascular homeostasis. It is re- quired for vasculogenesis and maintance of vascular permeability. Its expression and function is disturbed during inflammation.

In our prospective study we would like to described behaviour of these two cadherins during development and progression of atherosclerosis (on rabbit model af atherosclerosis) because changes in vascular permeability, neovasculogenesis and formation of lipid core are crucial for formation of atherosclerotic plaques.

\section{Acknowledgements}

The authors wish to thank Dr. B. Mánek, CSc. for revising the English text. This work was supported by MŠMT grant 111600002 .

\section{References}

1. Allport JR, Ding H, Collins T, Gerritsen ME, Luscinskas FW. Endothelial-dependent mechanisms regulate leukocyte transmigration: A process involving the proteasome and disruption of the vascular endothelial-cadherin complex at endothelial cell-to-cell junctions. J Exp Med 1997;186:517-27.

2. Andriopoulou P, Navarro P, Zanetti A, Lampugnani MG, Dejana E. Histamine induces tyrosine phosphorylation of endothelial cell-to-cell adherens junctions. Arterioscler Thromb Vasc Biol 1999;19:2286-97.

3. Asgeirsson KS , Jónasson JG, Tryggvadóttir L et al. Altered expression of E-cadherin in breast cancer: Patterns, mechanisms and clinical significance. Eur J Cancer 2000;36:1098-106.

4. Beavon IRG. The E-cadherin-catenin complex in tumour metastasis: Structure, function and regulation. Eur J Cancer 2000;36:1607-20.

5. Behrens J. Cadherins and catenins: Role in signal transduction and tumor progression. Cancer Metastasis Rev 1999;18:15-30.

6. Bendel-Stenzen MR, Gomperts M, Anderson R, Heasman J, Wylie CH. The role of cadherins during primordial germ cell migration and early gonad formation in the mouse. Mech Dev 2000;91:143-52.

7. Bobryshev YV, Lord RSA. Mapping of vascular dendritic cells in atherosclerotic arteries suggests their involvement in local immune- inflammatory reactions. Cardiovasc Res 1998;37:799-810.

8. Bobryshev YV, Cherian SM, Inder SJ, Lord SA. Neovascular expression of VEcadherin in human atherosclerotic arteries and its relation to intimal inflammation. Cardiovasc Res 1999;43:1003-17.

9. Bobryshev YV, Lord SA, Wanatabe T, Ikezawa T. The cell adhesion molecule Ecadherin is widely expressed in human atherosclerotic lesions. Cardiovasc Res 1998;40:191-205.

10. Breier G, Breviario F, Caveda L et al. Molecular cloning and expression of murine vascular endothelial-cadherin in early stage development of cardiovascular system. Blood 1996;87:630-41.

11. Breviario FL, Caveda M, Corada I et al. Functional properties of human vascular endothelial cadherin (7B4/cadherin-5) an endothelium-specific cadherin. Arterioscler Thromb Vasc 1995;15:1229-39.

12. Davies G, Jiang WG, Mason MD. E-cadherin and associated molecules in the invasion and progression of prostate cancer. Oncol Rep 1998;5:1567-76.

13. Dejana E, Bazzoni G, Lampugnani MG. Vascular endotelial (VE)-cadherin: Only an intercellular glue? Exp Cell Res 1999;252:13-9.

14. Dejana E, Corada M, Lampugnani MG. Endothelial cell-to cell junctions. FASEB J 1995;9:910-8.

15. Dejana E. Endothelial adherens junctions: Implications in the control of vascular permeability and angiogenesis. J Clin Invest 1996;98:1949-53.

16. El-Hariry I, Jordinson M, Lemoine N, Pignatelli M. Characterization of the Ecadherin-catenin complexes in pancreatic carcinoma cell lines. $J$ Pathol 1999;188:155-62.

17. Ghadimi BM, Behrens J, Hoffmann I, Haensch W, Birchmeier W, Schlag PM Immunohistological analysis of E-Cadherin, $\alpha$-, $\beta$ - and $\gamma$-catenin expression in colorectal cancer: Implications for cell adhesion and signaling. Eur J Cancer 1999;35:60-5

18. Hinck L, Nathke IS, Papkoff J, Nelson WJ. Dynamics of cadherin-catenin complex formation: Novel protein interactions and pathways of complex assembly. J Cell Biol 1994;125:1327-40.

19. Cell adhesion molecules: An overview. Cancer Invest 1998;16:176-82. JosephSilverstein J, Silverstein RL.

20. Lampugnani MG, Resnati M, Raiteri M et al. A novel endothelial-specific membrane protein is a marker of cell-cell contacts. J Cell Biol 1992;118:1511-22. 
21. Langille BL, Adamson SL. Relationship between blood flow direction and endothelial cell orientation at arterial branch sites in rabbits and mice. Circ Res 1981;48:481-8.

22. Mareckova Z, Heller S, Horky K. Cell adhesion molecules and their role in pathophysiological processes. Vnitr Lek 1999;45:232-7.

23. Muro del GX, Torregrosa A, Muňoz J et al. Prognostic value of the expression of E-cadherin and $\beta$-catenin in bladder cancer. Eur J Cancer 2000;36:357-62.

24. Navarro P, Ruco L, Dejana E. Differential Localization of VE- and N-cadherins in human endothelial cells: VE-Cadherin competes with N-cadherin for junctional localization. J Cell Biol 1998;140:1475-84.

25. Nollet F, Kools P, Van Roy F. Phylogenetic analysis of the cadherin superfamily allows identification of six major subfamilies besides several solitary members. J Mol Biol 2000;299:551-72.

26. Noria S, Cowan DB, Gotlieb AI, Langille L. Transient and steady-state effects of shear stress on endothelial cell adherens junctions. Circ Res 1999;85:504-14.

27. Petruzzelli L,Takami M, Humes HD. Structure and function of cell adhesion molecules. Am J Med 1999;106:467-76.

28. Rabiet MJ, Plantier JL, Rival Y, Genoux Y, Lampugnani MG, Dejana E. Thrombin-induced increase in endothelial permeability is associated with changes in cell-to-cell junction organization. Arterioscler Thromb Vasc Biol 1996;16:488-96.

29. Redies CH. Cadherins in the central nervous system. Prog Neurobiol 2000;61:611-48.

30. Reima I, Lehtonen E, Virtanen I, Flechon JE. The cytoskeleton and associated proteins during cleavage, compaction and blastocyst differentiation in the pig Differentiation 1993;54:35-45.

31. Rival Y, Del Maschio A, Rabiet MJ, Dejana E, Duperray A. Inhibition of platelet endothelial cell adhesion molecule-1 synthesis and leukocyte transmigration in endothelial cells by the combined action of TNF-alpha and IFN-gamma. J Immunol 1996:157:1233-41.

32. Romer LH, McLean NV, Yan HC, Daise M, Sun J, DeLisser HM. IFN-gamma and TNF-alpha induce redistribution of PECAM-1 (CD31) on human endothelial cells. J Immunol 1995;154:6582-92.

33. Romer LH, McLean NV, Yan HC, Daise M, Sun J, DeLisser HM. Interaction of neutrophils and endothelium in isolated coronary venules and arterioles. Am J Physiol 1995;268:490-8.

34. Shapiro L, Fannon AM, Kwong PD et al. Structural basis of cell-cell adhesion by cadherins. Nature 1995;374:327-37.

35. Shimamura K, Hirano S, McMahon, AP, Takeichi M. Wnt-1-dependent regulation of local E-cadherin and alpha N-catenin expression in the embryonic mouse brain. Development 1994;120:2225-34.
36. Shimamura K, Takeichi M. Local and transient expression of E-cadherin involved in mouse embryonic brain morphogenesis. Development 1992;116:1011-9.

37. Shino Y, Watanabe A, Yamada Y et al. Clinicopathologic evaluation of immunohistochemical E-cadherin expression in human gastric carcinomas. Cancer 1995; 76:2193-201

38. Steinberg MS, McNutt PM. Cadherins and their connections: Adhesion junctions have broader functions. Curr Opin Cell Biol 1999;11:554-60.

39. Taga M, Suginami H. Cell adhesion and reproduction. Horm Res 1998;50(supp 2):2-6

40. Takao S, Che X, Fukudome T et al. Downregulation of E-cadherin by antisense oligonucleotide enhances basement membrane invasion of pancreatic carcinoma cells. Hum Cell 2000;13:15-21.

41. Telo P, Lostaglio S, Dejana E. Structure of intercellular junctions in the endothelium. Therapie 1997;52:395-8.

42. Tinsley JH, Wu MH, Ma W, Taulman AC, Yuan SY. Activated neutrophils induce hyperpermeability and phosphorylation of adherens junction proteins in coronary venular endothelial cells. J Biol Chem 1999;274:24930-4

43. Vleminckx K, Kemler R. Cadherins and tissue formation: Integrating adhesion and signaling. Bioessays 1999;21:211-20.

44. Wong RK, Baldwin AL, Heimark RL. Cadherin-5 redistribution at sites of TNF- $\mathrm{c}$ and IFN- - -induced permeability in mesenteric venules. Am J Physiol 1999;276 (Heart Circ Physiol):736-48.

45. Yap AS, Niessen CM, Gumbiner BM. The juxtamembrane region of the cadherin cytoplasmic tail supports lateral clustering, adhesive strenghtening, and interaction with p120 ctn . J Cell Biol 1998;141:779-89.

Submitted April 2001.

Accepted November 2001.

Mgr. Petr Nachtigal,

Charles University in Prague,

Faculty of Pharmacy in Hradec Králové,

Department of Biological and Medical sciences,

Heyrovského 1203, 50005 Hradec Králové,

Czech Republic.

e-mail: nachti@faf.cuni.cz 